Marquette University

e-Publications@Marquette

Biomedical Engineering Faculty Research and

Publications

Biomedical Engineering, Department of

$1-1-2003$

\title{
Three-Dimensional Reconstruction Algorithm for a Reverse-Geometry Volumetric CT System With a Large-Array Scanned Source
}

Taly Gilat Schmidt

Marquette University, tal.gilat-schmidt@marquette.edu

Rebecca Fahrig

Stanford University

Norbert J. Pelc

Stanford University

Published version. Published as part of the proceedings of the conference, Medical Imaging 2003:

Physics of Medical Imaging, 2003: 103-111. DOI. (C) 2003 Society of Photo-Optical Instrumentation Engineers. One print or electronic copy may be made for personal use only. Systematic reproduction and distribution, duplication of any material in this paper for a fee or for commercial purposes, or modification of the content of the paper are prohibited. 


\title{
Three-dimensional reconstruction algorithm for a reverse geometry volumetric CT system with a large array scanned source
}

\author{
Taly Gilat ${ }^{a, b}$, Rebecca Fahrig ${ }^{a}$, Norbert J. Pelc ${ }^{a}$ \\ ${ }^{a}$ Department of Radiology, Stanford University, Stanford, CA 94305 \\ ${ }^{b}$ Department of Electrical Engineering, Stanford University, Stanford, CA 94305
}

\begin{abstract}
We have proposed a CT system design to rapidly produce volumetric images with negligible cone beam artifacts. The investigated system uses a large array scanned source with a sinaller array of fast detectors. The x-ray source is electronically steered across a $2 \mathrm{D}$ target every few milliseconds as the system rolates. The proposed reconstruction algorithm for this system is a modified $3 \mathrm{D}$ filtered backprojection method. The data are rebinned into $2 \mathrm{D}$ parallel ray projections, most of which are tilted with respect to the axis of rotation. Fach projection is filtered with a $2 \mathrm{D}$ kernel and backprojected onto the desired image matrix. To ensure adequate spatial resolution and low artifact level, we rebin the data onto an array that has sufficiently fine spatial and angular sampling. Due to finite sampling in the real system, some of the rebinned projections will be sparse, but we hypothesize that the large number of views will compensate for the data missing in a particular view. Preliminary results using simulated data with the expected discrete sampling of the source and detector arrays suggest that high resolution ( $<0.5 \mathrm{~mm}$ in all directions) images can be obtained in a single rotation with the proposed system and reconstruction algorithm.
\end{abstract}

Keywords: computed tomography (CT), volumetric CT, 3D reconstruction

\section{INTRODUCTION}

Faster scan times, large anatomic coverage with thin slices, and reduced motion artifacts have been made possible by the developrnent of multidetector computed tomography (MDCT) systems. The volume covered by current multidetector scanners is still relativcly small, for example these systems require many gantry rotations to acquire the full hearl volume. One logical extension of this approach is a system capable of imaging the entire volume in a single rotation, volumetric $\mathrm{CT}$ (VCT). As the acquired volume thickness increases, by increasing the number of detector rows or by using flat-panel digital x-ray detectors, so does the divergence angle of the $\mathrm{x}$-ray cone beam in the axial direction. The problem with VCT systems with a single circular orbit is that the acquired cone-beam data set is not sufficient for an exact reconstruction. ${ }^{1}$ For small cone angles the resulting artifacts are tolerable, but as the volume thickness increases, so does the severity of the artifarts.

We have proposed a VCT system that uses a large array scanned source and a smaller array of fast detectors. ln principle, the date set acquired by this reverse geometry system should be sufficient to prevent cone-bearn artifacts, as the source and detector arrays have the same axial extent.

The goal of this paper is to describe the proposed reconstruction algorithm for this system. The theoretical deiails of the algorithm will be presented, as well as the preliminary results.

\section{PROPOSED SYSTEM GEOMETRY}

The system wo are proposing to acquire a sufficient VCT data set is a reverse geometry system with a large array scanned souxce and a smaller array of detectors. The basic system geometry is illustrated in Fig. 1 .

The system is conceptnally similar to that used by NexRay, Inc. for their interventional cardiology Carm system. ${ }^{2}$ The proposed $\mathrm{x}$-ray source has an electron beam that is clectromagnetically steered across a transmission target. An array of collimator holes limits the resulting small area $x$-ray beam so it is aimed towards the detector. The detector is comprised of a smaller array of fast photon counting detectors. During 


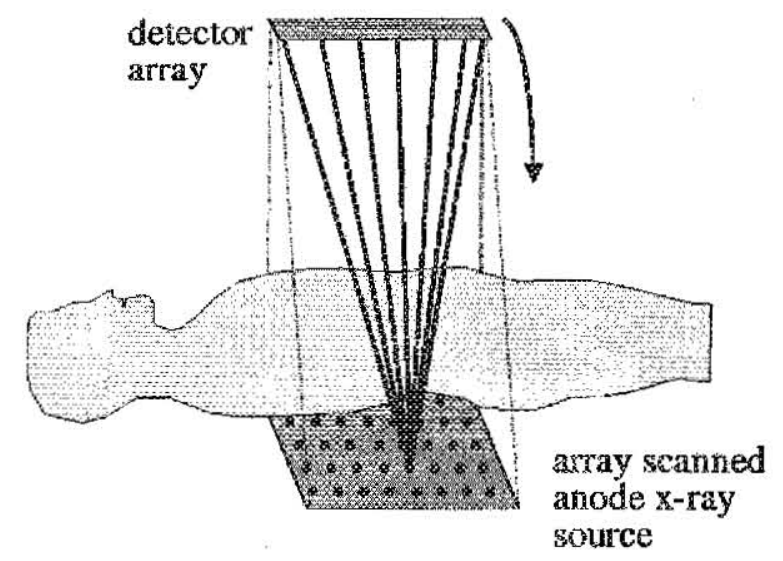

Figure 1. Proposcd system geometry showing the x-ray beam at one collinator position.

an acquisition, the $\mathrm{x}$-ray beam dwells on each source position (i.c., adjacent to each collimator hole) and then is steered to the next source position in the array. For each source position, the entire detector array is read ont producing a 2D divergent projection covering a fraction of the field of vicw.

In order to nse these components in a CT system, the source and detector would be mounted on a gantry and rotated around the patient.

T'he specifications for the preliminary $\mathrm{C} \Gamma$ geometry are summarized in Table 1.

Table 1. Specifications for proposed VCT geometry

\begin{tabular}{|l|l|}
\hline Source dimerisions (transverse $\mathrm{x}$ axial) & $50 \mathrm{~cm} \times 15 \mathrm{~cm}$ \\
\hline Number of source locations & $200 \times 60$ spots \\
\hline Detector dimensions (transverse $\mathrm{x}$ axial) & $5 \mathrm{~cm} \times 15 \mathrm{~cm}$ \\
\hline Number of detector locations & $48 \times 144$ elements \\
\hline Dwell time per source location & $1 \mu \mathrm{s}$ \\
\hline Move time between successive source locations & $0.28 \mu \mathrm{s}$ \\
\hline
\end{tabular}

\section{RECONSTRUCTYON ALGORTTHM}

The proposed 3D reconstruction algorithm can be broken down into a rebinning step followed by filtered backprojection. The basic idea, of the algorithm is to view the data not as they are acquired, but instead as a set of $2 \mathrm{D}$ parallel ray projections. Among the acquired rays are a set of parallel rays that are perpendicular to the axis of rotation. These "in-plane" ravs are equivalent to those collected in a normal CT scan. In addition to these in-plane rays, the acquired data set also contains cross plane rays that are tilted with respect to the axis of rotation. This concept is illustrated in Fig. 2. The reconstruction algorithm first rebins the acquired data into a set of $2 \mathrm{D}$ parallel ray projections at numerous tilt angles, and then uses these $2 \mathrm{D}$ projections to reconstruct a volume. Because the source and detector sampling is finite, some of these rebinned projections may be sparse. However, because of the large number sampled views, we hypothesize that missing data in one view can be compensated by nearby projections.

This reconstruction schene is motivated by the Fourier domain interpretation of $2 \mathrm{D}$ parallel ray projections. This analysis, based on the Central Slice Theorem, will be described in the filtcred backprojection section. It,

Proc. of SPIE Vol. 5030 


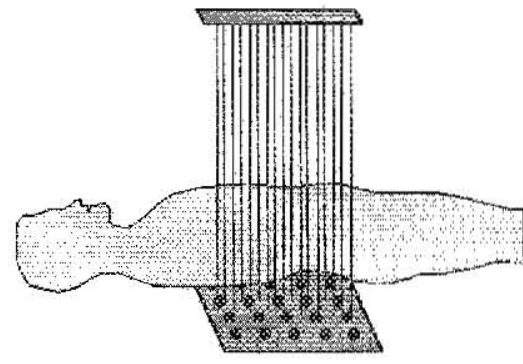

(a) An in-planc parallel ray projection

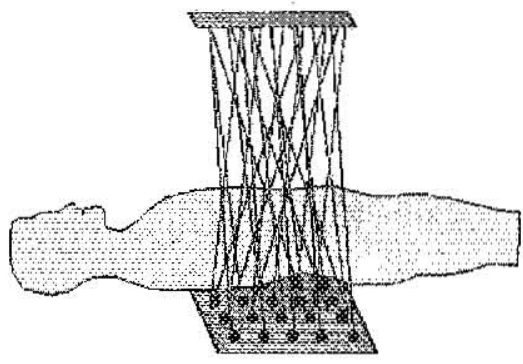

(b) Cross-plane rays

Figure 2. Rebinining acquired data into 2D parallel ray projections.

should also be noted that the rebimed data set resembles the data acquired in multi-ring Positron Emission Tomography (PET). Therefore, techniques already developed for PET reconstruction can be utilized.

The following sections describe the theory involved in the two steps of the proposed reconstruction incthod, and also discuss the preliminary implementation.

\subsection{Rebinning algorithm}

Each ray from a source location to a detector location is described by four parameters, the rotation angle, $\dot{\phi}$, the colatitude or tilt angle (the angle from the axis of rotation), $\theta$, and two distance measures, $\rho_{i p}$ and $\rho_{o p}$, describing the location of the line from the centrul ray. These parameters are illustrated in Fig. 3 . The two distance parameters could be combined into one distance parameter, but are kept separate in this discussion.

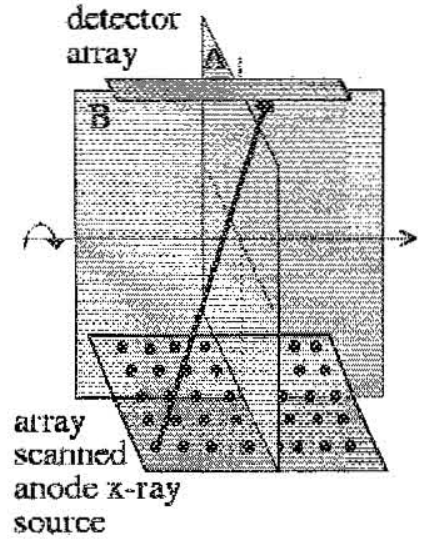

(a) A ray from the source to the detector.

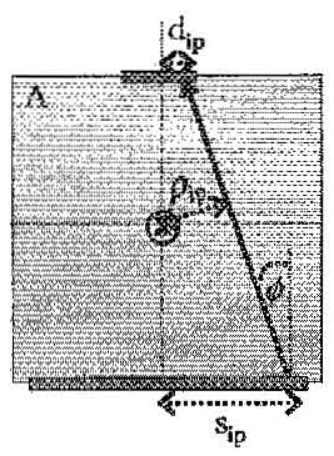

(b) The projection of the ray onto plane $A$.

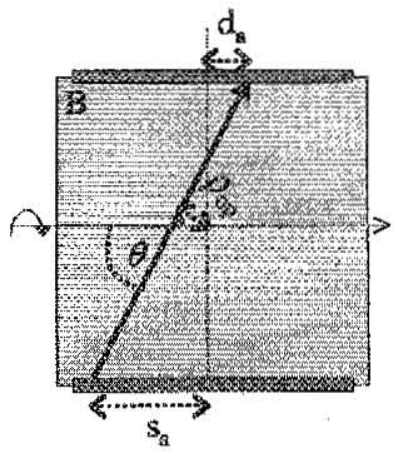

(c) The projection of the ray onto plare $B$.

Figure 3. Determining the four geometry parameters describing a ray in 3D space.

The four parameters can be determined geometrically. The rotation angle, $\phi$, and in-plane distance, $\rho_{i p}$, 
depend on the in-plane source location, $s_{i p}$, and detector location, $d_{i p}$. As illustrated in Fig. 3(b), these two parameters can be found using the following equations.

$$
\begin{gathered}
\dot{\psi}=\arctan \left(\frac{s_{i p}-d_{i p}}{S D D}\right) \\
\phi=\psi+\phi_{\text {gantry }}
\end{gathered}
$$

where $\psi$ is the rotation angle of the ray without guntry rotation, and SDD is the source to detector distance. To find the total rotation, the gantry rotation, $\phi_{\text {gantry }}$ is added to $\psi$ as shown in Eq. 2 . The in-plane distance is

$$
\rho_{i p}=d_{i p} * \cos (\psi)+D I D * \sin (\psi)
$$

where $M I D$ is the detector to isocenter distance. As shown in Eq. $3, \rho_{i p}$ is independent of gantry rotation.

Similarly, $\theta$ and $\rho_{o p}$ depend on the axial source and detector positions, $s_{a}$ and $d_{a}$ respectively as shown in Fig. 3(c), and can be calculated using the following equations.

$$
\begin{gathered}
\theta=\frac{\pi}{2}-\arctan \left(\frac{s_{a}-d_{a}}{S D D}\right) \\
\rho_{o p}=d_{a} * \sin (\theta)+D I D * \cos (\theta)
\end{gathered}
$$

The goal of the rebinning algorithm is to take ench ray and place it into a $2 \mathrm{D}$ parallel ray projection. T'he two angle parameters, $\phi$ and $\theta$, determine the gantry rotation angle and the gantry tilt angle of the corresponding projection, and the two distance parameters determine the ray's location within the projection. Therefore, these four parameters are sufficient for reorganizing the data into 2D parallel ray projections. However, in a discrete implementation with a reasonable number of projections, especially projections that are equally spaced in each of the four parancters, ncarby rays must be bimned together using some form of interpolation or gridding.

To better understand the rebinuing algorithm, it is useful to visualize the data in Radon space. For 2D reconstruction with $1 \mathrm{D}$ projections, such as those acquired by conventional single slice $\mathrm{CT}$ systems, each acquired ray can be described by two parameters, the projection angle $\phi$ and the perpendicular distance of the ray to the axis of rotation, $\rho$. For single slice CT systoms, Radon space is two dimensional, with $\rho$ and $\phi$ as the two coordinate axes. Therefore, each ray acquired in a $1 D$ projection samples one point in the two dinensional Radon space. Each parallel projection samples one horizontal lize in 21 Radon space. In a single slice fan-beam system, each fan-beani samples a tilted linc in Radon space.

For our proposed system geometry, each ray is described by two angles and two distances, and thus is represented by a four dimensional Radon space. Each ray sanples one point in $4 \mathrm{D}$ Radon space, but the sample points from all the input rays are not distributed eveniy. The rebinning algorithm must convert the nonuniformly sampled AD Radon space data into uniformly spaced samples

'The problem of resampling nonuniform data onto a uniform grid has come up in many different fields, and much work has been done to solve it. We are using the gridding approach."

The first step in the gridding algorithm is to determine a bin width or kernel width for each of the four geonetry parameters. Our current, implementation is output-grid-driven. That is, for each output grid point in our uniformly spaced ID Radon space, the algorithm finds the input data points that fall within the $4 \mathrm{D}$ bin. Each input data point is weighted based on its distance to the grid point, where the weight is determined by a chosen $4 D$ kcrnel shape. At the output grid point, the weighted values of all the contributing data points are accumulated as are the sum of all the weights.

One important step in the rebinuing algorithm is to compensate for the nonumiform sampling density of the original data points. This can be done by pre-wcighting the data according to the sampling density of the input data points, by post-weighting the output data based on the total deposited weights at each grid point, 
or by using a combination of both methods. In our preliminary implementation, we are using post-weighting compensation and have also been investigating more accuraie pre-weighting methods.

The important design parameters in the gridding algorithm are the bin widths, kernel shape, and output grid sampling density. For application in MRI reconstruction, the effect of each of these parameters on the rebimned data has been described in detail. ${ }^{4}$ For the prelininary VCT implementation of this algorithun, these parameters were chosen experimentally. For computaiional simplicity, the $4 \mathrm{D}$ kernel was designed as four separable Hanning window kernels in each dimension. The output grid spacing was chosen as the largest spacing that provided acceptably low artifacts. The bin widths were chosen to trade off the blurring caused by large bin widths while reducing the occurrence of emply output grid points which are caused by small bin widths.

\subsection{Filtered backprojection}

The key to accurate reconstruction for filtered backprojection is in the filter design. In the spatial domain, the filtering step can be seen as correcting the impulse response of the backprojection process. That is, the generally negative tails of the reconstruction kernel exactly cancel the positive tails (blurring) that results if a band limited impulse is backprojected.

Having the data organized into $2 \mathrm{D}$ parallel ray projections provides a useful method for designing the filter in Fourier space using the Central Slice 'L'heorem, which states that a 2D parallel ray projection of a 3D object samples the Fourier transform of the object along the plane perpendicular to the projection direction. So as mumerous 2 D projections are acquired, the Fourier transform of the object is sampled along the corresponding plancs. As a result, 3D frequency space is not sampled uniformly, with certain regions sampled more than others. It can also be shown that the Fourier transform of the backprojection of a single $2 \mathrm{D}$ projection into a 3D volume is non-zero only on the same plane that was sampled by that projection. ${ }^{5}$

The role of the reconstruction filters is to weight the frequency content of each projection so that, when they arc all superimposed, the 3D Fourier transform of the object is properly reconstructed. In our approach, the filter applied to each projection is the inverse of the density of measurements in frequency space on the plane sampled by that projection.

An analytical solution for this filter has been derived for $2 \mathrm{D}$ parallel ray projections equally spaced over a range of tilt angles ${ }^{6}$ and will be stated without proof below.

The 2D filter for a 2D parallel ray projection at a colatitude angle $\theta$ is

$$
G_{\theta}\left(k_{u}, k_{v}\right)=\frac{W(k)}{D_{\theta}(k, \alpha)}
$$

where $k_{u}$ and $k_{v}$ are the coordinates of the $2 \mathrm{D}$ Fourier transform of the projection, we define

$$
\begin{gathered}
k=\sqrt{k_{u}^{2}+k_{v}^{2}} \\
\alpha=\arccos \left(\frac{k_{v} \sin \theta}{k}\right)
\end{gathered}
$$

and $W(\mathrm{k})$ is a window function used to control the impulse response. $D_{\theta}$ is the density of measurements on the plane in frequency space that is sampled by the projection. For our case, we assume that the projections are continuously and uniformly distributed between $\phi$ cqual to zero and $2 \pi$ and colatitude angle betwcen $\theta_{\min }$ and $\pi / 2$, where $\theta_{\min }$ is the colatitude angle of the most oblique projection. This is a reasonable assumption if the distance between adjacent projections is small in both angular directions. The resulting $D_{\theta}$, without proof, is

$$
D_{\theta}(k, \alpha)=\frac{M \arcsin \left(\frac{\cos \left(\theta^{\prime}\right)}{\sin (\alpha)}\right)}{\pi k \cos \left(\theta_{\min }\right)}
$$

where $\mathrm{M}$ is the total number of projections and $\theta^{\prime}$ is defined as

$$
\theta^{\prime}=\max \left(\theta_{\min }, \frac{\pi}{2}-\alpha\right)
$$


Using the expression for $D_{0}$, we can write the equation for the $2 \mathrm{D}$ filter as

$$
C_{\theta}\left(k_{u}, k_{v}\right)=\frac{\pi k \cos \left(\theta_{\min }\right)}{M \arcsin \left(\frac{\cos \left(H^{\prime}\right)}{\sin (\alpha)}\right)} * W(k)
$$

As can be seen, the filter depends on the colatitude or tilt angle of a given projection, but is the same for all projections at that colatitude angle.

To summarize the filtered backprojection step, for each rebinned 2D parallel ray projection, the 2D Fourier transform is calculated. The transform is then multiplied by the 2D filter described in Eq. 11. Finally the filtered projection is three dimensionally backprojected into the output volume.

\section{METHODS}

The 3D filtered backprojection algorithm has been inplemented for reconstructing a volume from 2D parallel ray projections at multiple tilt angles. This algorithru has been tested with simulated ideal parallel ray projections and found to have artifacts at levels below the CT noise floor.

The AD Radon space rebinning algorithm has becu implemented and is currently boing tested.

To explore the inuge quality performance of the rebinuing algorithn, a simpler $2 \mathrm{D}$ version was inplementod to reconstruct 2D slices from the in-plane sampling of the source and detector illustrated in Fig. 4 (i.e. an inplane geometry). This algorithm takes the rays connecting one source row to one detector row and rebins them into 10 parallel ray projections. Standard filtered backprojection is used to reconstrnct the in-plane slice from these 10 projections. This in-plane reconstruction algorithm provides a method to test the in-plane resolution of the system, and also to investigate the effects of the rebinning algorithm on the output image quality.

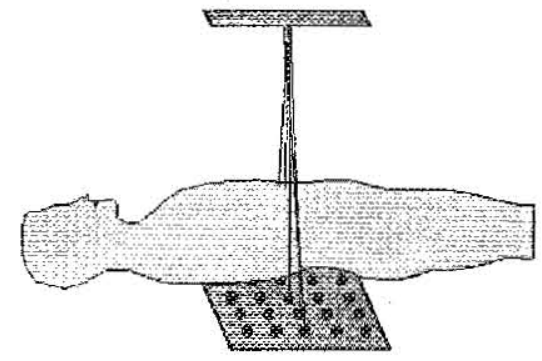

Figure 4. the in-plane sampling is the rays connecting one source row and one detector row collected during a full rotation aboul the object.

The following section briefly describes the results obtained using the in-plane reconstruction algorithm. The two experiments described below use the simulated in-plane sampling of the available NexRay source and detector. The simulated source row had 100 elements over $25 \mathrm{~cm}$, and the simulated detector row had 48 elements over $5 \mathrm{~cm}$. Seventy-one projections over a 360 degree gantry rotation (each comprised of rays connecting all source locations in one source row with all the detector elements in one detector row) were simulated with the in-plane geometry, as this number of projections was found to sufficiently sample the 2D Radon space for the field of view (FOV) used. The projections were then rebinned into 1000 parallel ray projections with an output detector pitch of $1 / 8 \mathrm{~mm}$. For comparison, ideal $1 D$ parallel ray projections wore also generated. The ideal parallel ray projections had the same Radon space sampling as the rebimed projections. 'I'he simulated detector aperture and source focal spot blurring were kept constant for both simulations, with the focal spot modeled as a $0.8 \mathrm{~mm}$ by $0.8 \mathrm{mun}$ rect function and the detector aperture modeled as a $1 \mathrm{~mm}$ by $1 \mathrm{~mm}$ rect function. Standard fittered backprojection was then used to reconstruct the image. 
For the rebinned in-plane projections, a second rcconstruction was perforwed where the backprojection filter was modified to undo the blurring caused by the rebinning step. The correction was based on the Fourier transform of the $\rho$ gridding kernel. 'This deapodization function was thresholded so as not to greatly amplify any artifacts or noise.

ln all cuses, the reconstructed FOV was $7 \times 7 \mathrm{~cm}$ with a pixel size of $1 / 8 \times 1 / 8 \mathrm{~mm}$.

\section{RESULTS}

\subsection{In-plane resolution}

The in-plaxe reconstruction algorithm has made it possible to investigate the resolution capabilities of the system. The in-plane MTF was calculated by simulating a small sphere at iso-center with radius $1 / 16 \mathrm{~mm}$. Fig. 5 compares the MIE for the three simulated cases: the in-plane sampling, ideal parallel ray projections, and in-plane sampling with correction for the blurring from the gridding step. It is important to note that for the first two cases the backprojection filter was windowed with a Haming window; filters with higher gain at high spatial frequencies could be used and would yield higher spatial resolution. Fig. 5 shows that slight blurring is introduced by the rebinning algorithm, but that most of this blurring can be recovered with the modified roconstmiction filter. For the in-plane sampling, the $10 \%$ point is approximately $14 \mathrm{~cm}^{-1}$ without the deapodization, and is approxinately $18 \mathrm{~cm}^{-1}$ with the correction.

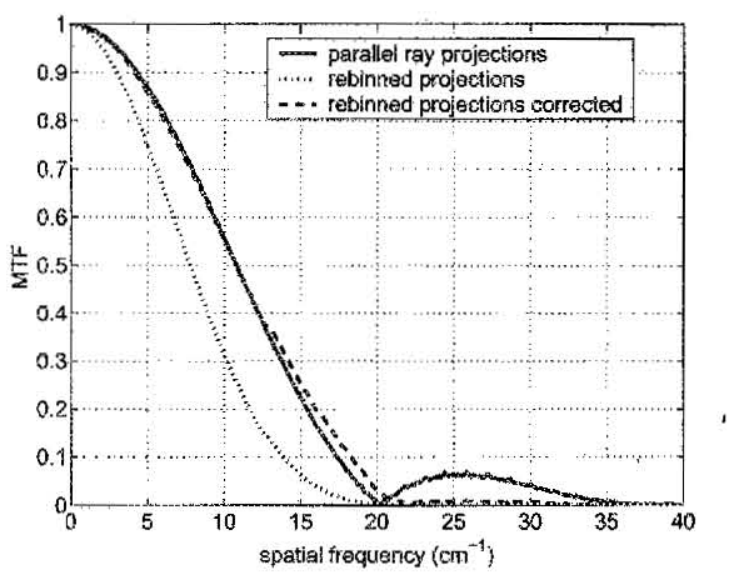

Figure 5. MTF comparison for idcal parallel ray projections, rebinned in-plane projections, and rebinuce in-plano projections with correctod backprojection filter.

A resolution phantom was also simulated using small sphere objects. 'The resolution parterns ranged from $0.7 \mathrm{~mm}(7.1 \mathrm{lp} / \mathrm{cm})$ in the upper right hand corner, to $0.4 \mathrm{~mm}(12.5 \mathrm{lp} / \mathrm{cm})$ in the lower right hand corner. Fig. 6 shows the inages resulting from the three reconstructions.

Comparing the images, the blurring caused by the rabinning algorithm is noticeable, bui using the modified filter does improve the resolution. Iooking at the corrected in-plane reconstruction, Fig. 6(c), some resolution loss can be seen towards the cdge of the $\mathrm{FOV}$, which is likely due to the residual blurring in the $\phi$ direction introduced by the rebinning algorithm. An additional deapodization stcp, before fltered backprojection, could be inplemented to reduce this. Despite the blurring caused by the rebinning step, for all three reconstructions, the 0.4 num paltern can be rosolved.

\subsection{Rebinning algorithm artifacts}

'To explore the accuracy of the rebinning algorithm, projections were simulated through an off-center uniform sphere of water. 


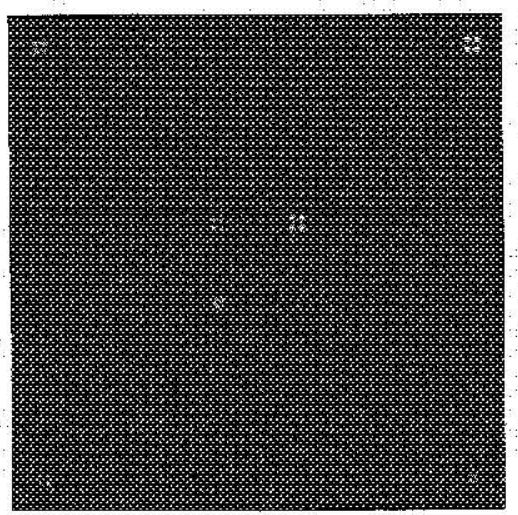

(a) in-plane sampling

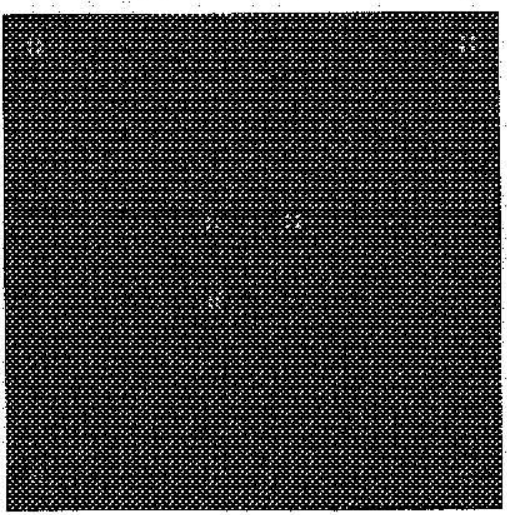

(b) idcal parallel ray

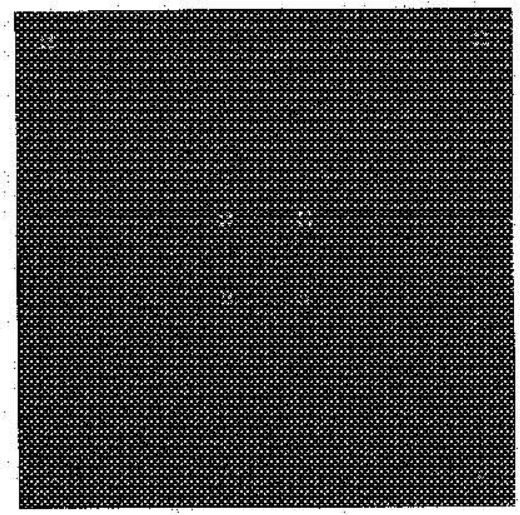

(c) in-plane sampling with corrected backprojection filter

Figure 6. Sinulated resolution phantom. Tested frequeneics are, counter-clockwise from the uppor right hand quadrant, $0.7 \mathrm{~mm}, 0.6 \mathrm{mmm}, 0.5 \mathrm{mim}, 0.4 \mathrm{~mm}$.

The sphere had a radius of $2.5 \mathrm{~cm}$ and was centered at $(0.5 \mathrm{~cm}, 0.5 \mathrm{~cm})$. Fig. 7 shows images reconstructed using the rebinned in-plane projections, the ideal paralld ray projections, and the rebinned in-plane rays with correction. The inges are windowed to the lovel of $0 \mathrm{HL}$ and a widh of $+/-1 \mathrm{HU}$ (i.e., values -1 and +1 HU are mapped to black and white, respectively). Comparing the images, the artifact level within the water sphere is similar for all images and is below $1 \mathrm{HU}$. 'The ideal and corrected reconstructions have ringing artifacts at the edge of the sphere that are not present in the image with the unmodified rebinued rays due to bluming introduced by the rebinning algorithm.

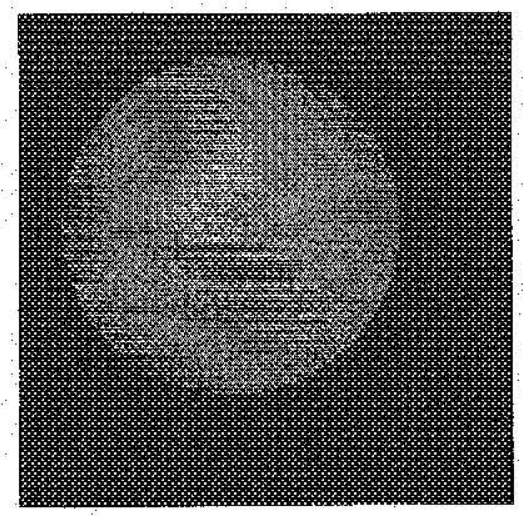

(a) in-plane sasnpling

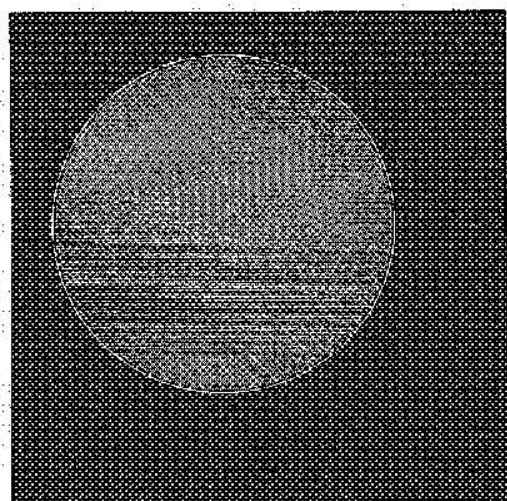

(b) ideal parallel ray

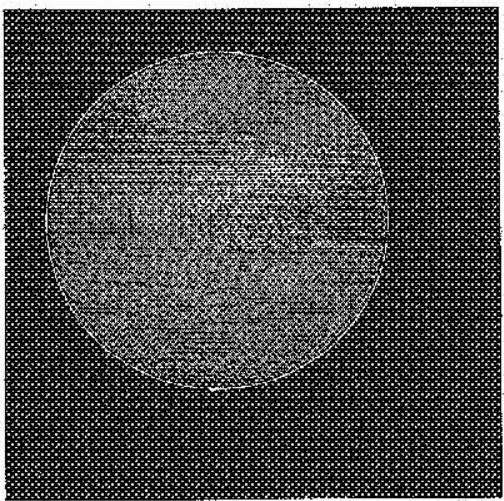

(c) in-plane sampling with corrected backprojection filter

Figure 7 . Simulated water sphere. These inages were windowed to a lcvel of 0 HU and a width of $+/-1$ HU to show artifacts in the sphere.

Fig. 8 shows the three images windowed to a level of $-1000 \mathrm{HU}$ with a window width of $+/-1$ HU to show 


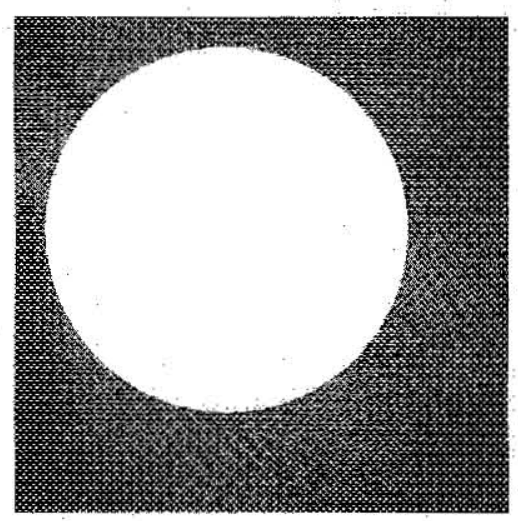

(a) itr-plane sampling

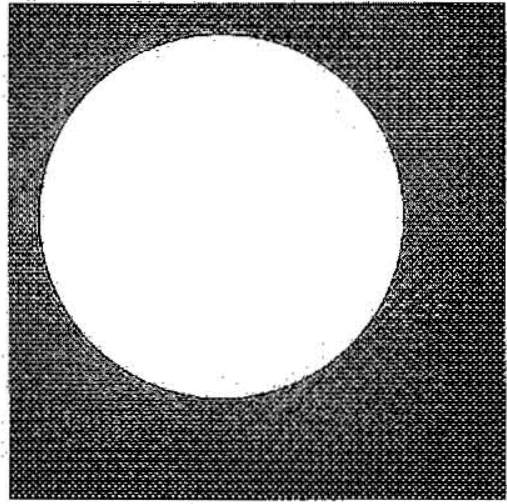

(b) idcal parallel ray

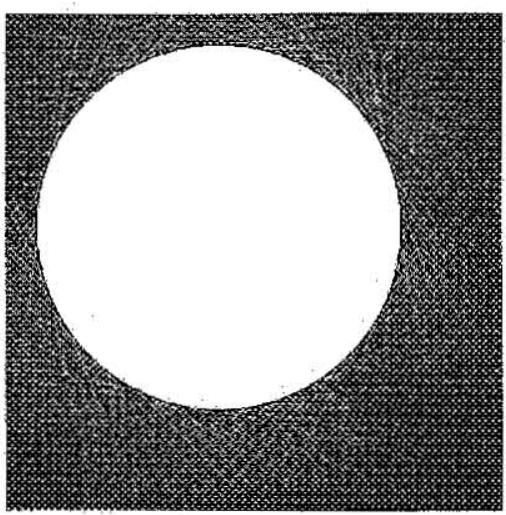

(c) in-plane sampling with corrected backprojection filter

Figure 8. Sirudated water sphere. These images were windowed to a level of $-1000 \mathrm{HC}$ and a width of $+/-1 \mathrm{HU}$ to slow wrifacis outside the object

artifacts in air. The in-plane sampling has more view aliasing artifacts than the ideal parallel ray geometry, and these artifacts are amplified by the modified backprojection filter used in Fig. 8(c). Despite this amplification, the artifacts are on the order of $1 \mathrm{HU}$ and thus are acceptably low.

\section{CONCLUSIONS}

Although the complete reconstruction algorithm has not yet been fully tested, preliminary results suggest that the proposed algorithm is feasible for the reverse geometry VCT system. Results using the in-plane reconstruction algorithrn show that the robimning step introduces uo significant artifacts and only slight blurring, most of which can be recovered using a corected reconstruction filter. Experiments using the in-plane reconstruction algorithm also demonstrate an in-plane resolution better than $0.5 \mathrm{~mm}$.

\section{ACKNOWLEDGMENTS}

This work is supported by GE Medical Systems. 'The authors would like to thank Edward Solomon of NexRay, Inc. for helpful discussions.

\section{REFERENCES}

1. B. D. Smith, "Cone-beam tomography: recent advances and a tutorial review," Optical Engineering 29 , pp. 524-534, 1990.

2. E. G. Solomon, B. P. Wilfley, M. S. Van Lysel, A. W. Joseph, and J. A. Hcanue, "Scanniug-beam digital X-ray (SBDX) system for cardiac angiography," in Medical Imaging 1999: Physics of Medical Imaging. Proc. SPIE 3659, pp. 246-257, 1999.

3. J. O'Sullivan, "A fasi sinc function gridding algorithn for Fourier inversion in computer tomograpy," IEEE Tran. Med. Imaging 4, pp. 200-207, 1985.

4. J. 1. Jackson, C. II. Meyer, D. G. Nishinnura, and A. Macovski, "Selection of a convolution function for Fourier inversion using gridding," IEEE Trans. Med. Imaging 10, pp. 473-478, 1991.

5. N. J. Pelc and D. A. Chesler, "Utilization of cross-plane rays for three-dimensional reconstruction by filtered back-projection," J. Comput. Assist. Tomogr. 3, pp. 385-395, 1979.

6. N. J. Pels; " $\Lambda$ generalized filtered backprojection algorithm for three dimensional reconstruction,". PhD dissertation, Harvard University, Boston, MA, 1979. 